\title{
Knee kinematic curve representation and application to knee pathology classification
}

\author{
Badreddine Ben Nouma ${ }^{1}$, Amar Mitiche ${ }^{1}$, Youssef Ouakrim*2, Neila Mezghani ${ }^{2,3}$ \\ ${ }^{1}$ INRS - Énergie, matériaux et télécommunications, Montreal, Quebec, Canada \\ ${ }^{2}$ LICEF Research Center, TELUQ University, Montreal, Quebec, Canada \\ ${ }^{3}$ Laboratoire de recherche en imagerie et orthopédie, CRCHUM, Montreal, Quebec, Canada
}

Received: February 28, 2018

DOI: $10.5430 /$ jbei.v4n1p32
Accepted: April 10, 2018

URL: https://doi.org/10.5430/jbei.v4n1p32

\begin{abstract}
This study investigates a variational method to determine the most representative shape of a set of knee kinematic curves with application to knee pathology classification. Although they provide essential information for pathology classification, knee kinematic curves are characterized by high intra-class variability and outliers are often present. As a result, a set of several measurement curves are acquired of any single individual which are then averaged before their use for pathology classification. Rather than using the average of an individual's recorded measurement curves, this method determines a better representative curve by first correcting the data to account for outliers occurrence and class variability using a variational method. The correction is performed by simultaneous minimization of a set of objective functions, one for each curve in the measurement set, and consisting of a weighed sum of two terms: a data term of conformity of the corrected curve to the given curve, and a regularization term of proximity of the corrected curve to the mean of all the corrected curves to inhibit the influence of outliers in the set. Validation tests were performed to discriminate between knee osteoarthritis data (OA) and non-OA data. Using a support vector machine, the classification accuracy with the proposed representation was $86 \%$, with $81 \%$ sensitivity and $90 \%$ specificity, compared to $83 \%$ accuracy for the standard representation by average, with $76 \%$ sensitivity and $90 \%$ specificity. The representation has also been tested within the OA category to distinguish the femero-tibial patholgy from the femero-patellar, giving $76 \%$ accuracy, with $76 \%$ sensitivity and $76 \%$ specificity, compared to $69 \%$ accuracy, with $62 \%$ sensitivity and $76 \%$ specificity. These significant improvements by the proposed method warrant its further investigation by application to other biomedical engineering pattern classification problems and datasets.
\end{abstract}

Key Words: Knee kinematic data curves, Knee pathology classification, Particle swarm optimization

\section{INTRODUCTION}

Three-dimensional (3D) knee kinematic data, measured during a walking session on a dedicated treadmill, consist of three types of curves describing the temporal variation during locomotion of the three fundamental angles of knee rotation: the flexion/extension angle, with respect to the sagittal plane, abduction/adduction angle, which is measured with respect to the frontal plane, and the internal/external angle, with respect to the transverse plane (as illustrated in Figure 1). The three angles of interest contain information essential to knee movement analysis for pathology diagnostic and can guide treatment plans. ${ }^{[1-4]}$

*Correspondence: Youssef Ouakrim; Email: oua.youssef@ gmail.com; Address: LICEF Research Center, TELUQ University, Montreal, Quebec, Canada. 

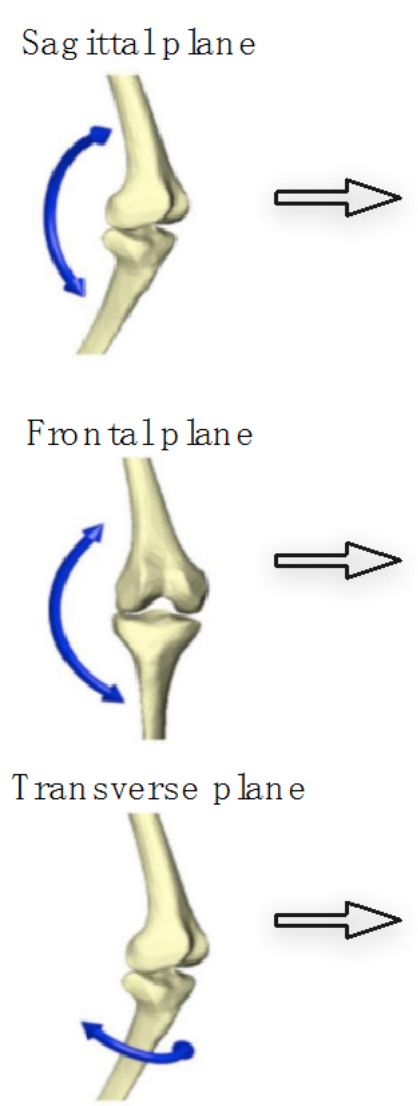

(a) Flexion/extension

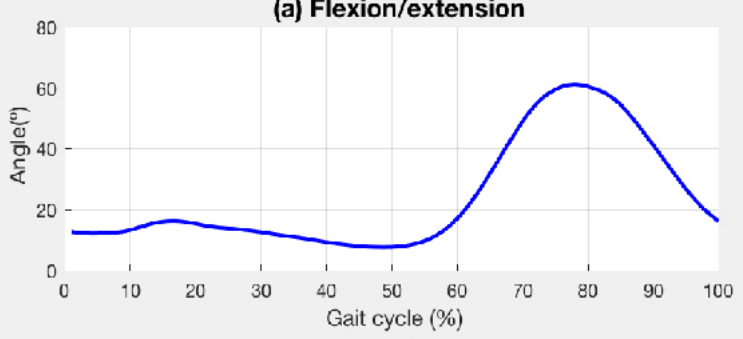

(b) Abduction/adduction

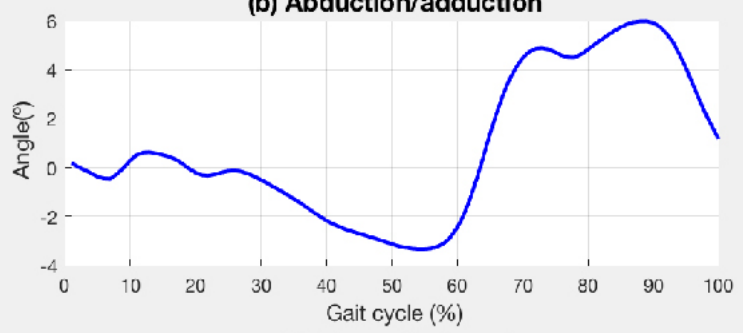

(c) Internal/external

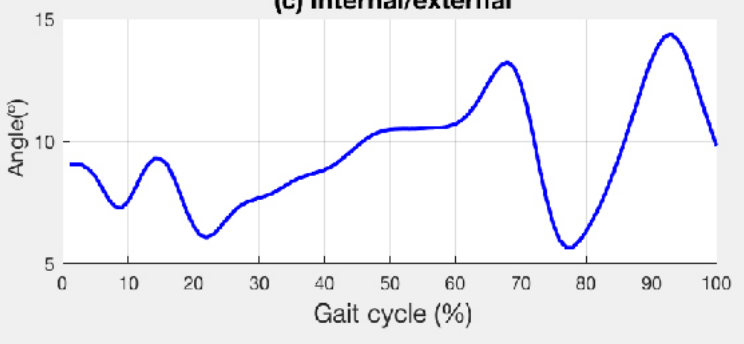

Figure 1. Three-dimensional (3D) knee kinematic data in the three fundamental planes: flexion/extension (sagittal plane), abduction/adduction (frontal plane), and tibial internal/external rotation (transverse plane)

Although the measurements for these angles can be recorded accuratly in clinical settings, ${ }^{[5]}$ they suffer of high variabilies. Indeed, the participant's angle variation pattern in time changes in general, sometimes significantly, from one cycle to another during locomotion, because of the uneven nature of a walker's cadence. This results in 3D kinematic curves of high variability, ${ }^{[6]}$ and possibly affected by outliers $^{[7]}$ as illustrated in Figure 2.

To account for the high variability, and for the occurrence of outliers, current studies use, for each participant, the average over the several curves, typically twenty to thirty, recorded from the participant during an extended walking task or the average over the curves that maximize the inter-class correlation index. ${ }^{[8,9]}$ This average curve becomes the participant's representative curve, to be used in subsequent investigations and analysis, for instance to assist in the diagnosis of knee pathologies such as affections by the osteoarthritis disease. ${ }^{[10]}$ Because a simple average curve is notoriously sensitive to high variability in the data it summarizes, particularly when outliers occur, this study investigates whether one can find a better representative curve in the sense that it yields higher knee pathology classification accuracy. To this effect, it investigates a variational method which determines the most representative shape of a participant's kinematic data curves by averaging these after a correction that inhibits the impact of high variability and outlier occurrence. This correction is performed by minimizing simultaneous objective functions, one for each curve, consisting of a weighed sum of two terms: a data term for the corrected curve conformity to the original, recorded data curve, and a regularization term of proximity of the corrected curve to the mean of the corrected set of curves to inhibit the influence of outliers and high variability. Minimization of the simultaneous objective functions is done efficiently by particle swarm optimization, a method which, contrary to gradient descent, is generally robust to the presence of outlying data. ${ }^{[11]}$ We have applied the resulting variational algorithm to knee osteoarthritis pathology classification in several experiments using distinct test data sets. The effectiveness of the algorithm was tested in terms of sensibility, specificity, and classification accuracy criteria. Comparative results show that the method improves significantly on existing schemes.

The remainder of this paper is organized as follows: Section 2 describes the method including, the knee kinematic data 
collection, the objective function and its minimization by described in Section 3 and the conclusion is given in Section particle swarm optimization. The experimental results are 4

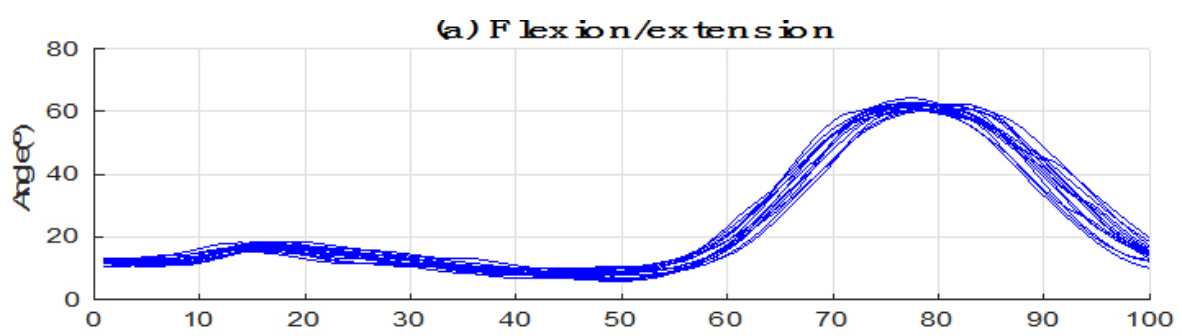

(b) A bduction/adduction

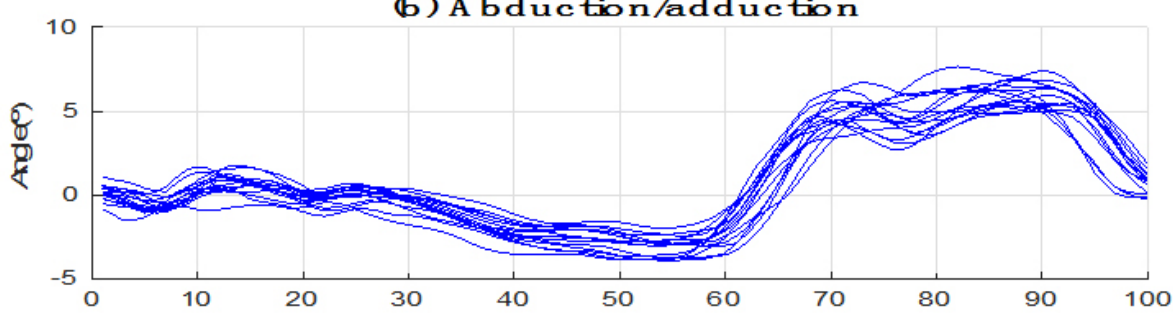

(c) In ternal/external
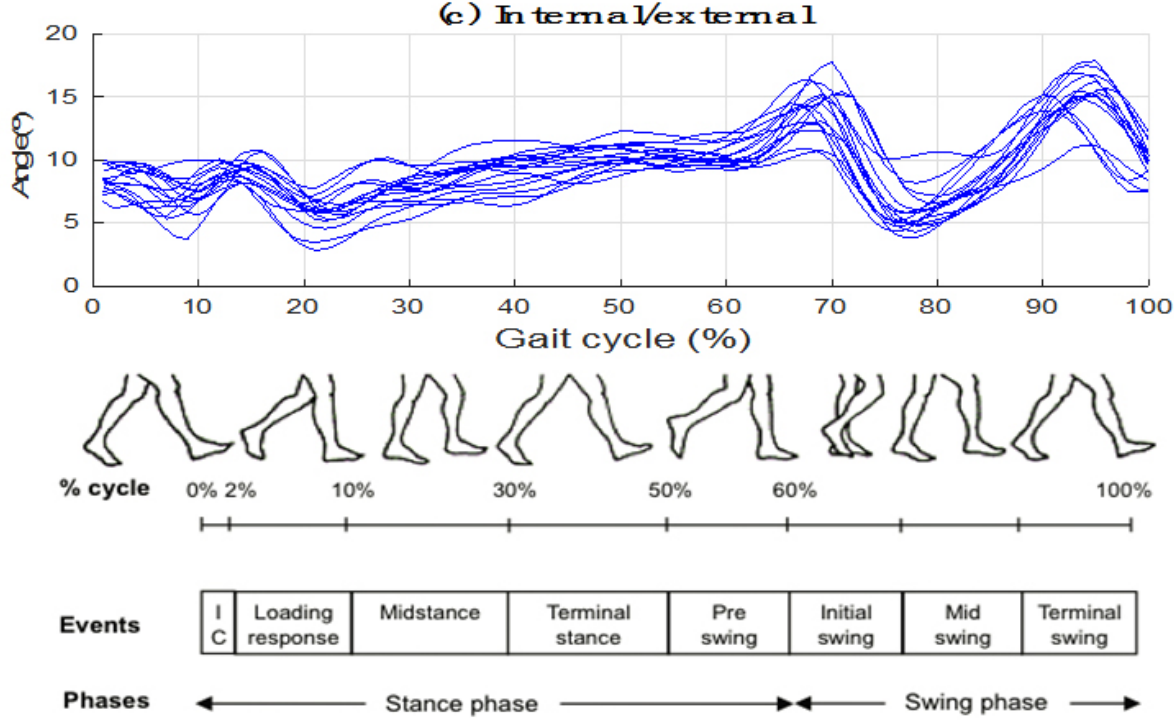

Figure 2. A family of a participant's 25 knee kinematic data curves: (a) Flexion/extension, (b) Abduction/adduction, and (c) Internal/external rotation. Each curve was interpolated and re-sampled for 100 evenly spaced points (1\% to $100 \%$ of a gait cycle, where $1 \%$ corresponds to the initial contact (IC) and 100\% to the end of the swing phase)

\section{METHOD}

\subsection{Knee kinematic data collection}

The kinematic data were recorded, from each participant, with respect to the frontal, sagittal, and transverse planes during a conventional treadmill walking task at a self-selected comfortable speed. This was possible thanks to a knee marker attachment system, the KneeKG ${ }^{T M}$ system (see Figure 3), which was installed on the participant's knee to record the 3D kinematics during $25 \mathrm{sec} .{ }^{[5]}$ For each participant, a set of twenty or thirty knee kinematic data curves is collected. These curves are re-sampled at $1 \%$ to $100 \%$ of the gait cycle, therefore giving 100 measurement points. The re-sampled curves are then corrected to obtain the most representative curve. ${ }^{[12]}$

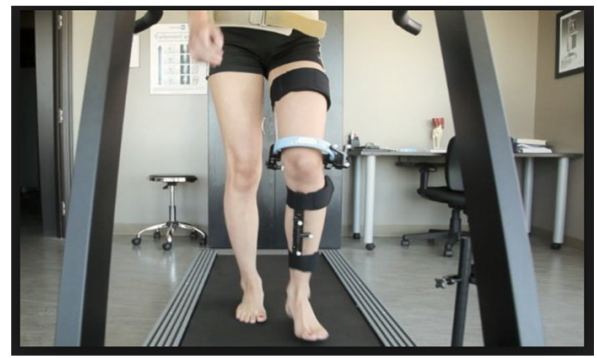

Figure 3. Knee Kinematic data acquisition system 


\subsection{Knee kinematic data sets}

The data come from knee osteoarthritis patients and healthy individuals. The data from the osteoarthritis patients (OA) is illustrated in Figure 4 which displays a set representation of the different categories of the disease considered in the experiments we performed. Osteoarthritis data composes the superset (OA) of the disease. In this superset, we distinguish two subsets: data from femero-tibial deseased joint category, abbreviated FT, data from femero-patellar diseased joint category, abbreviated FR. The union of FR and FT categories does not cover the OA superset since there are other types of knee joint diseases. The complement of the OA category is the N-OA category, which represents any knee condition, including healthy, that is not osteoarthritis.

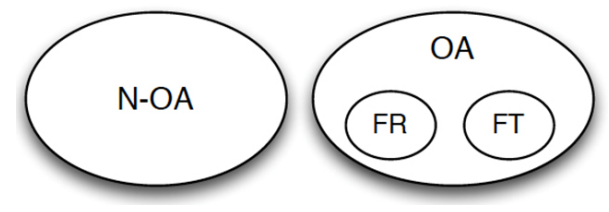

Figure 4. A set representation of the different disease categories

Two kinematic datasets are used to test the proposed method. The first dataset (which we call DS1) is composed of data from the two categories, FR and FT just described. Only a small sample of 21 measurements is available for each category. The second used dataset (DS2) contains measurements from 21 knee osteoarthritis patients (called OA) and 21 participants without knee osteoarthritis (which we call N-OA). The demographic characteristics of DS1 are summarized in Table 1 and for DS2 is in Table 2. Values are mean and standard deviation (SD) of each characteristic.

Table 1. Demographic characteristics for the three categories of twenty-one participants each: FR and FT of the first data set (DS1: FR/FT dataset)

\begin{tabular}{lcc}
\hline Characteristics & FR & FT \\
\hline Age (years) & $46.1 \pm 11.7$ & $59.5 \pm 10.1$ \\
Height $(\mathrm{m})$ & $1.71 \pm 0.07$ & $1.66 \pm 0.09$ \\
Weight $(\mathrm{kg})$ & $82.9 \pm 20.7$ & $76.2 \pm 11.2$ \\
BMI $\left(\mathrm{kg} / \mathrm{m}^{2}\right)$ & $28.3 \pm 7.1$ & $27.04 \pm 3.9$ \\
Men/group $(\%)$ & 45 & 38 \\
\hline
\end{tabular}

Note. Values are mean \pm standard deviation

\subsection{Representative curve identification}

We applied the variational method developed in this study to real-world knee kinematic data curves, and then used the class representative curves it determines in classification experiments involving knee osteoarthritis pathologies. Sensitivity, specificity, and classification accuracy criteria were Published by Sciedu Press used to evaluate the descriptive potency of the class representative curves. Here following are detailed descriptions of the data sets and the validation tests.

Table 2. Demographic characteristics for the two categories of twenty-one participants each: OA and N-OA of the second data set (DS2: OA/N-OA dataset)

\begin{tabular}{lcc}
\hline Characteristics & OA & N-OA \\
\hline Age (y) & $63 \pm 8$ & $64 \pm 9.2$ \\
Height $(\mathrm{m})$ & $1.72 \pm 0.1$ & $1.73 \pm 0.1$ \\
Weight $(\mathrm{kg})$ & $82.9 \pm 17.2$ & $72.1 \pm 12.9$ \\
BMI $\left(\mathrm{kg} / \mathrm{m}^{2}\right)$ & $29.1 \pm 5.5$ & $24.0 \pm 2.4$ \\
Men/group (\%) & 28.5 & 57.1 \\
\hline
\end{tabular}

Note. Values are mean \pm standard deviation

\subsubsection{Objective function}

Let $\left\{\mathbf{d}_{i}, i=1, \cdots, n\right\}$ be a family of $n$ knee kinematic data curves, measured for a given participant, and $\left\{\mathbf{c}_{i}, i=\right.$ $1, \cdots, n\}$ a corresponding family of corrected curves. As described earlier, a curve measures the temporal variation of one of the fundamental angles of knee rotation, and the purpose of corrections is to account for intra-class high variability and outlier occurrence which characterize the knee kinematic data.

Let $\mu=\frac{1}{n} \sum_{j=1}^{n} \mathbf{c}_{j}$ be the mean of corrected curves $\left\{\mathbf{c}_{i}, i=1, \cdots, n\right\}$. The mean of the corrected curves becomes the participant's representative curve, to be used subsequently for classification.

In practice, curves are vectors of a number of points on their graph. The problem is to determine a family of corrected curves which minimize the following system of simultaneous functions:

$$
\begin{aligned}
& \mathcal{E}_{1}\left(\mathbf{c}_{1}, \mathbf{c}_{2}, \ldots, \mathbf{c}_{n}\right)=\varepsilon\left\|\mathbf{c}_{1}-\mathbf{d}_{1}\right\|+(1-\varepsilon)\left\|\mathbf{c}_{1}-\mu\right\| \\
& \mathcal{E}_{2}\left(\mathbf{c}_{1}, \mathbf{c}_{2}, \ldots, \mathbf{c}_{n}\right)=\varepsilon\left\|\mathbf{c}_{2}-\mathbf{d}_{2}\right\|+(1-\varepsilon)\left\|\mathbf{c}_{2}-\mu\right\| \\
& \ldots \\
& \mathcal{E}_{n}\left(\mathbf{c}_{1}, \mathbf{c}_{2}, \ldots, \mathbf{c}_{n}\right)=\varepsilon\left\|\mathbf{c}_{n}-\mathbf{d}_{n}\right\|+(1-\varepsilon)\left\|\mathbf{c}_{n}-\mu\right\|,
\end{aligned}
$$

where $\|$.$\| designates the Euclidean norm, and \varepsilon$ is a real parameter to weigh the relative contribution of the two terms in the expression of functions $\varepsilon_{i}, i=1, \cdots, n$. For each index, $i=1, \cdots, n$, the first term of $\varepsilon_{i}$ is a data term, to measure the conformity of the corrected curve to the originally measured data curve, and the second term is a regularization term, to draw the corrected curve toward the mean of the corrected curves.

\subsubsection{Optimization}

From a general point of view and top level organization, the iterative algorithm to minimize system (1) is as follows: 
Algorithm 1: Optimization algorithm to minimize System (1)

- Initialize $\mathbf{c}_{i}, i=1, \ldots, n$

- Initialize $\mu$

- for $k=1, k \max$

- begin

- for $i=1, \ldots n$

* begin

* Minimize $\varepsilon_{i}$ with respect to $\mathbf{c}_{i}$

$*$ end

- Update $\mu$

- end

- end

At each iteration $k$, of a total number kmax of iterations, the mean $\mu$, which serves subsequently to represent the class of the data curves of interest, is computed using the curves $\mathbf{c}_{i}$ of the previous iteration $k-1$. Therefore, the minimization of $\varepsilon_{i}$ at each step does not involve the mean curve as a variable. The gradient descent necessary conditions for a minimum of $\varepsilon_{i}$ with respect to correction curves $\mathbf{c}_{i}$ can be written eas- ily. However, 3D kinematic data is notoriously prone to the presence of outliers. For this kind of data, particle swarm optimization (PSO) ${ }^{[13-15]}$ is much more efficient because, contrary to gradient descent, it is robust to the presence of outlying data. ${ }^{[7]}$ PSO is a stochastic optimization algorithm which uses a set of evolving solutions, which are variables of the type of a data curve, called particles, which it iteratively modifies toward a minimizer of system (1). The standard version of the PSO algorithm is detailed in ${ }^{[16]}$ and computer programs are available from several different sources.

Given the task we want to perform, namely to correct a set of $n$ curves to minimize (1), we will use PSO in an opportunistic way by having the correction curves $\left.\left\{\mathbf{c}_{i}\right\}\right|_{1} ^{n}$ be the particles (Algorithm 2). We can, conveniently, initialize the evolving correction curves to the given data curves $\left.\left\{\mathbf{d}_{i}\right\}\right|_{1} ^{n}$ and then evolve them according to a common PSO procedure, ${ }^{[1]}$ using the following update rule, for $i=1, \cdots, n$

$$
\mathbf{c}_{i}(k)=\mathbf{c}_{i}(k-1)+\mathbf{v}_{i}(k)
$$

where (particle velocity) $\mathbf{v}_{i}$ is given by:

$$
\mathbf{v}_{i}(k)=w \mathbf{v}_{i}(k-1)+\alpha\left(\mathbf{b}_{i}(k-1)-\mathbf{c}_{i}(k-1)\right)+\beta\left(\mathbf{g}(k-1)-\mathbf{c}_{i}(k-1)\right)
$$

in which $w$ is a constant parameter; $\alpha$ and $\beta$ are random being constant parameters; $\mathbf{b}_{i}, \mathbf{g}$ are given by: numbers in the range $\left[0, z_{1}\right]$ and $\left[0, z_{2}\right]$ respectively, $z_{1}, z_{2}$

$$
\begin{aligned}
& \mathbf{b}_{i}(k)=\arg \min _{\mathbf{x} \in\left\{\mathbf{b}_{i}(k), \mathbf{c}_{i}(k)\right\}} \mathcal{E}_{i}\left(\mathbf{c}_{1}(k), \ldots, \mathbf{c}_{i-1}(k), \mathbf{x}, \mathbf{c}_{i+1}(k), \ldots, \mathbf{c}_{n}(k)\right) \\
& \mathbf{g}(k)=\arg \min _{\mathbf{x} \in\left\{\mathbf{b}_{i}(k), i=1, \ldots n,\right\}} \mathcal{E}_{i}\left(\mathbf{c}_{1}(k), \ldots, \mathbf{c}_{i-1}(k), \mathbf{x}, \mathbf{c}_{i+1}(k), \ldots, \mathbf{c}_{n}(k)\right)
\end{aligned}
$$

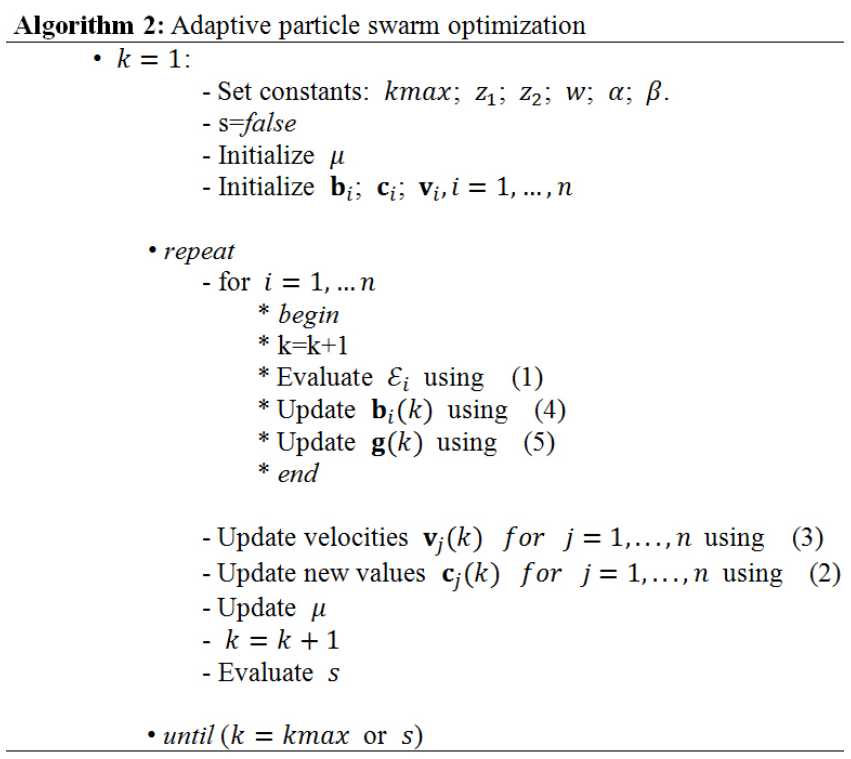

In these expressions, $\mathbf{b}_{i}$ and $\mathbf{g}$ respectively, the best performing particle $i$ so far (i.e., at the current iteration $k$ ) and best overall particle. These operations are embedded in the following pseudo program, where $s$ is a boolean variable to be true when the corrected curves have changed too little at the current iteration, according to some threshold in Algorithm 2 .

\subsection{Data curve representation validation}

The descriptive potency of the proposed knee kinematic curve representation will be tested via classification experiments involving knee osteoarthritis pathologies. The evaluated criteria are sensitivity, specificity, and classification accuracy. We used four different classifiers: K-nearest neighbors (KNN), Naive Bayes (NB), Linear discriminant analysis (LDA), and a Support vector machine (SVM). 


\section{Classification accuracy, sensitivity, and specificity}

The classification accuracy $(\tau)$, sensitivity $(S e)$, and specificity $(S p)$ are defined as follows:

$$
\begin{gathered}
S e=\frac{T P}{T P+F N} \\
S p=\frac{T N}{T N+F P} \\
\tau=\frac{T P+T N}{T P+T N+F P+F N^{\prime}}
\end{gathered}
$$

where $T P$ is the number of true positives, $T N$ is the number of true negatives, $F P$ is the number of false positives, and $F N$ is the number of false negatives. For OA and N-OA data example, TP is the number of OA participants correctly classified as OA patients and $F P$ is the number of non-OA participants correctly classified as non-OA patients.

The classification results were evaluated by leave-one-out cross validation, as is generally done in classification accuracy evaluation, consisting of using one sample for validation, and the others for learning, for all possible choices of the test sample, and finally averaging the corresponding classification accuracies.

\subsection{Comparisons}

We compared the performance of the curve representation determined by our method to the benchmark representation by the average curve used in the litterature. ${ }^{[7]}$ The classification experiments were done using the four different classifiers, i.e., K-nearest neighbors (KNN), Naive Bayes (NB), Linear discriminant analysis (LDA), and a Support vector machine (SVM). We tested with the two datasets described earlier, DS1 and DS2. The details and the results are given subsequently.

\section{RESULTS AND DISCUSSION}

The data was collected from different participants, each described by a set of twenty or thirty measurement repetitions. For each participant, the set of curves are corrected using the proposed variational method. The mean of the corrected curves is then taken to be the participant's representative curve. The tests were conducted using the data sets DS1, composed of $21 \mathrm{FR}$ and $21 \mathrm{FT}$ patients, and DS2, which contains measurements from 21 knee OA patients and 21 N-OA participants.

Figure 5 illustrates, for one participant from DS1, twenty such measured curves (blue curves) and their representative shape (red curve) as determined by the proposed variational method. Figure 6 shows on the same graph, for visual comparison, an example of the proposed representation and the benchmark average curve, for each of the three planes of the reference system associated with a knee. The representative data curve determined by the proposed method is drawn in red and the benchmark average data curve in blue. We can see that the difference between the two curves is at places significant.

Figure 5 shows the high variability and the occurrence of outliers that the data curves of one participant, in blue, exhibit. Since an average is sensitive to high variability and outlying measurements, this example foretells the problems that might occur in classification when a subject's data curves are summarized by their average. The proposed variational method determines the most representative shape of a participant's data curves by averaging these after a correction that inhibits the impact of high variability and outlier occurrence. One can note in Figure 6 that the proposed representation is smoother at places than the average curve.

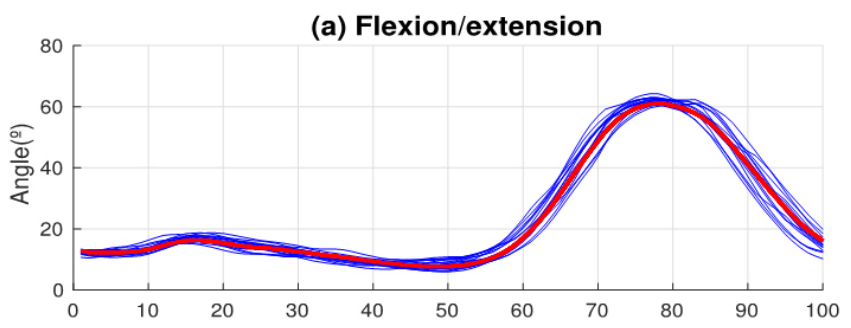

(b) Abduction/adduction

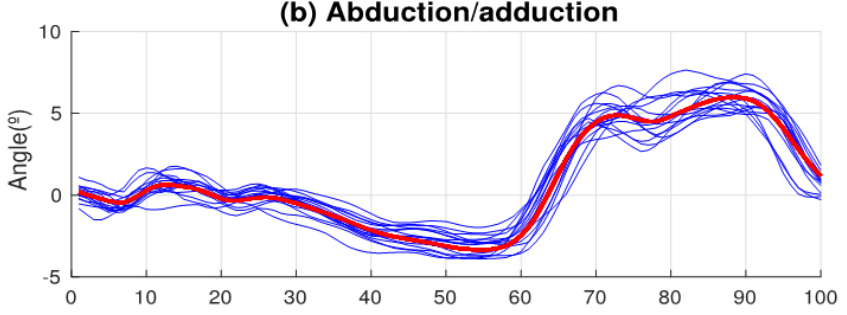

(c) Internal/external

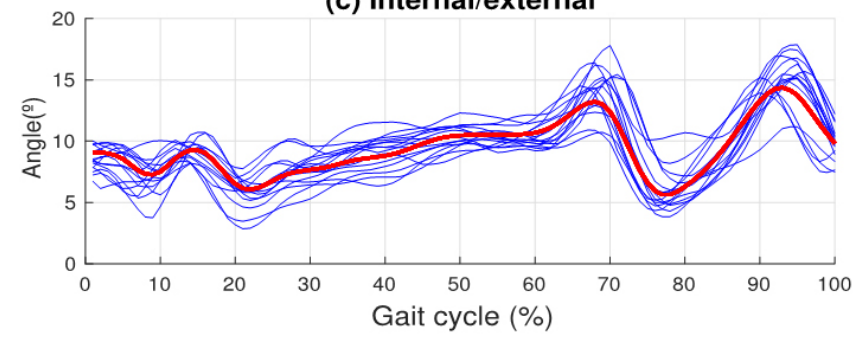

Figure 5. Knee kinematic data curves from DS1 of a particular participant: (a) Flexion/extension, (b) Abduction/adduction, and (c) Internal/external rotation. The blue curves show the family of measured kinematic data and the red curve corresponds to the computed most representative shape using the variational method 

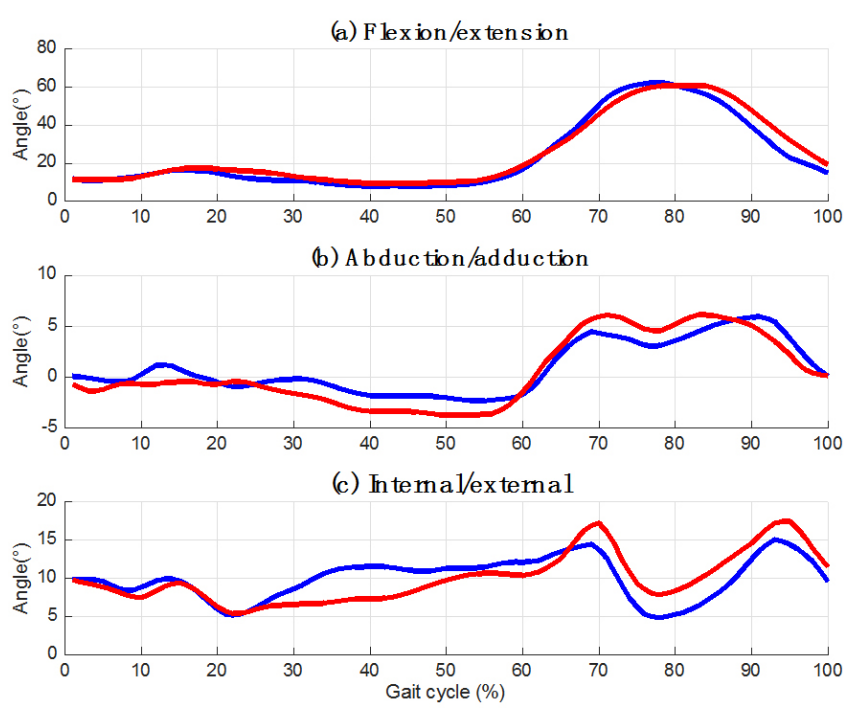

Figure 6. Knee kinematic data curves for a particular participant from DS1. The blue curve represents the average of the measured kinematic data and the red curve corresponds to the representative shape obtained by the proposed variational method
As explained in Section 2.5, the effectiveness of the representative shape is evaluated in terms of classification performance and tested using four classification methods: KNN, NB, DA, and SVM. Algorithms are implemented using Matlab software (Mathworks). These classification experiments use FR and FT datasets, DS1, to distinguish FT from FR, and tha dataset DS2, of OA and N-OA data, to distinguish OA from N-OA.

Table 3 summarizes the results of the four classification methods and presents a comparison in terms of sensitivity, specificity, and accuracy. These results compare the criteria evaluated using the proposed method of data representation (variational method) and the benchmark average curve. In practically all cases, the classification rates are higher with the proposed method of representation. SVM classification gives the best results for both representations. The classification accuracy is $76 \%$ on DS1 and $86 \%$ on DS2 for the proposed representation, against, respectively, $69 \%$ and $76 \%$ for the representation by averaging. The corresponding sensitivities and specificities can be read off (see Table 3). In general, the proposed representation improves significantly on the standard representation by averaging.

Table 3. Sensitivity (Se), specificity (Sp), and classification rates $(\tau)$ of the different classifiers with respect to the two representation methods in the DS1 (FR/FT dataset) and in the DS2 (OA/N-OA dataset)

\begin{tabular}{|c|c|c|c|c|c|}
\hline \multirow[b]{2}{*}{ Classifiers } & \multirow[b]{2}{*}{ Criteria } & \multicolumn{2}{|c|}{ DS1: FR/FT dataset } & \multicolumn{2}{|c|}{ DS2: OA/N-OA dataset } \\
\hline & & $\begin{array}{l}\text { Average method } \\
(\%)\end{array}$ & $\begin{array}{l}\text { Variational method } \\
\text { (\%) }\end{array}$ & $\begin{array}{l}\text { Average method } \\
\text { (\%) }\end{array}$ & $\begin{array}{l}\text { Variational method } \\
\text { (\%) }\end{array}$ \\
\hline \multirow{3}{*}{ KNN } & Se & 67 & 95 & 71 & 95 \\
\hline & Sp & 67 & 62 & 81 & 62 \\
\hline & $\tau$ & 67 & 78 & 76 & 78 \\
\hline \multirow{3}{*}{ Naive Bayes } & Se & 76 & 81 & 67 & 71 \\
\hline & Sp & 48 & 71 & 81 & 81 \\
\hline & $\tau$ & 62 & 76 & 74 & 76 \\
\hline \multirow{3}{*}{$\begin{array}{l}\text { Discriminant } \\
\text { analysis }\end{array}$} & Se & 59 & 74 & 78 & 79 \\
\hline & Sp & 67 & 79 & 71 & 74 \\
\hline & $\tau$ & 62 & 76 & 73 & 76 \\
\hline \multirow{3}{*}{ SVM } & Se & 62 & 76 & 76 & 81 \\
\hline & Sp & 76 & 76 & 90 & 90 \\
\hline & $\tau$ & 69 & 76 & 83 & 86 \\
\hline
\end{tabular}

\section{Conclusion}

This study investigated a variational method to determine the most representative shape of a family of curves and applied it to kinematic data curves in the context of knee pathologies classification. Rather than using the average of an individual's recorded measurement curves, this method determined a better representative curve by first correcting the data to account for outlier occurrence and class variability. The correction was performed by simultaneous minimization of a set of objective functions, one for each curve in the measurement set, and consisting of a weighed sum of two terms: a data term of conformity of the corrected curve to the given curve, and a regularization term of proximity of the corrected curve to the mean of all the corrected curves to inhibit the influence 
of outliers in the set. We applied the method to elicit the representation shape of knee 3D movement and used it in knee pathology classification. In the comparative experimentation, several benchmark classifiers were used, namely K-nearest neighbors (KNN), Naive Bayes (NB), Linear discriminant analysis (LDA), and Support vector machines (SVM), and their performance was compared when they used the representative data curve determined by the proposed variational method and a direct average of a participant's measurement curves. The experiments were performed on two different data sets collected from healthy individuals and knee osteoarthritis patients with distinguished knee diseases. The results showed that the proposed representation outperformed the benchmark representation by averaging, and warrant further investigation by applications to other biomedical engineering pattern classification problems and datasets.

\section{REFERENCES}

[1] Duhamel A, Bourriez J, Devos P, et al. Kinematic and kinetic patterns in human gait: variability and compensating effects. Gait $\&$ posture. 2004; 20(2): 204-212. PMid:15336292. https ://doi.org/10.1 016/j.gaitpost.2003.09.010

[2] Gaudreault N, Mezghani N, Turcot K, et al. Effects of physiotherapy treatment on knee osteoarthritis gait data using principal component analysis. Clinical Biomechanics. 2010.

[3] Hunter DJ. Focusing osteoarthritis management on modifiable risk factors and future therapeutic prospects. Therapeutic Advances in Musculoskeletal Disease. 2009; 1: 35-47. PMid:22870426. https: //doi.org/10.1177/1759720X09342132

[4] Mezghani N, Ouakrim Y, Fuentes A, et al. Mechanical biomarkers of medial compartment knee osteoarthritis diagnosis and severity grading: Discovery phase. Journal of Biomechanics. 2017; 52(1): 106-112. PMid:28088304. https://doi.org/10.1016/j.jbio mech.2016.12.022

[5] Lustig S, Magnussen RA, Cheze L, et al. The kneekg system: a review of the literature. Knee Surgery, Sports Traumatology, Arthroscopy. 2012; 20(4): 633-638. PMid:22215077. https ://doi.org/10.1 007/s00167-011-1867-4

[6] Mezghani N, Husse S, Boivin K, et al. Identification of knee frontal plane kinematic patterns in normal gait by principal component analysis. Journal of Mechanics in Medicine and Biology. 2008; 13(3): r1230-1232.

[7] Mechmeche I, Mitiche A, Ouakrim Y, et al. Data correction to determine a representative pattern of a set of $3 \mathrm{~d}$ knee kinematic measurements. In 2016 38th Annual International Conference of the IEEE Engineering in Medicine and Biology Society (EMBC); 2016. p. 884-887.
[8] Tooke JE, et al. The effects of intravenous insulin infusion on skin microcirculatory flow in Type 1 diabetes. International Journal of Microcirculation, Clinical and Experimental/sponsored by the European Society for Microcirculation. 1984; 4(1): 69-83.

[9] Fleiss JL. Reliability of measurement. The design and analysis of clinical experiments; 1986. p. 1-32.

[10] Winter DA. Kinematic and kinetic patterns in human gait: variability and compensating effects. Human Movement Science. 1984; 3(1-2): $51-76$.

[11] Jamian JJ, Abdullah MN, Mokhlis H, et al. Global particle swarm optimization for high dimension numerical functions analysis. Journal of Applied Mathematics. 2014.

[12] Mezghani N, Fuentes A, Gaudreault N, et al. Identification of knee frontal plane kinematic patterns in normal gait by principal component analysis. Journal of Mechanics in Medicine and Biology. 2013; 13(3): 1350026. https://doi.org/10.1142/S0219519413500 267

[13] Eberhart RC, Hu XH. Human tremor analysis using particle swarm optimization. In Evolutionary Computation, 1999. CEC 99. Proceedings of the 1999 Congress on, volume 3. IEEE, 1999.

[14] Kenndy J, Eberhart RC. Particle swarm optimization. In Proceedings of IEEE International Conference on Neural Networks. 1995; 4: 1942-1948. https://doi .org/10.1109/ICNN . 1995. 488968

[15] Kennedy J, Mendes R. Population structure and particle swarm performance. 2002.

[16] Thida M, Eng HL, Monekosso DN, et al. A particle swarm optimisation algorithm with interactive swarms for tracking multiple targets. Applied Soft Computing. 2013; 13(6): 3106-3117. https://doi.org/10.1016/j.asoc. 2012.05.019

[17] Kennedy J, Eberhart RC, Shi Y. Swarm intelligence, morgan kaufmann publishers. Inc., San Francisco, CA, 2001. 